\title{
Advances in Laplace Type Integral Transforms with Applications
}

\author{
A. Aghili* and H. Zeinali \\ Department of Applied Mathematics, Faculty of Mathematical Sciences, University of Guilan, P.O.Box, 1841, \\ Rasht, Iran; arman.aghili@gmail.com, homa_zeinaly@yahoo.com
}

\begin{abstract}
In this study, the authors implemented one and two dimensional Laplace transform, Stieltjes and Post-Widder transforms to evaluate certain integrals and series. In the last section, certain fractional wave equation is solved via joint LaplaceFourier sine transform.

Illustrative examples are also provided. The result reveals that the integral transform method performs extremely well in terms of simplicity and efficiency.
\end{abstract}

Mathematics Subject Classification 2010: 26A33; 34A08; 34K37; 35R11.

Keywords: Bessel's Functions, Mathieu Series, Post-Widder transform, Two Dimensional Laplace Transform, Stieltjes Transform

\section{Primary Concepts}

DEFINITION 1.1 Laplace transform of the function $f(t)$ is defined as follows

$$
L\{f(t) ; t \rightarrow s\}=\int_{0}^{\infty} e^{-s t} f(t) d t:=F(s)
$$

If $L\{f(t)\}=F(s)$, then $L^{-1}\{F(s)\}$ is given by

$$
f(t)=\frac{1}{2 \pi i} \int_{c-i \infty}^{c+i \infty} e^{s t} F(s) d s
$$

where $F(s)$ is analytic in the region $\operatorname{Re}(s)>c$.

Definition 1.2 For an arbitrary real number $\alpha>0$ $(n-1 \leq \alpha<n, n \in N)$ Caputo fractional derivative ${ }^{9}$

$$
{ }_{a}^{C} D_{t}^{\alpha} f(t)=\frac{1}{\Gamma(n-\alpha)} \int_{a}^{t} \frac{f^{(n)}(x)}{(t-x)^{\alpha-n+1}} d x
$$

Definition 1.3 The Dirac delta function is defined by some authors as the function having the properties

$$
\begin{aligned}
& 1-\quad \delta(x-a)= \begin{cases}0 & x=a \\
+\infty & x \neq a\end{cases} \\
& 2-\quad \int_{-\infty}^{+\infty} \delta(x-a) \phi(x) d x=\phi(a) . \quad a \in R \\
& 3-\quad \phi(t) \delta(t-a)=\phi(a) \delta(t-a)
\end{aligned}
$$

Where $\phi(x)$ is any continuous and bounded function.

Problem 1.4 Evaluate inverse Laplace transform of the function

$$
F(s)=\frac{e^{-\lambda s^{\alpha}}}{s^{\beta}} ; \quad 0<\alpha<1, \quad 0 \leq \beta<1 .
$$

Solution. Integrating over the close contour $\Gamma$ we have

$$
\begin{aligned}
\frac{1}{2 \pi i} \int_{c-i T}^{c+i T} \frac{e^{-\lambda s^{\alpha}}}{s^{\beta}} e^{s t} d s & +\frac{1}{2 \pi i} \int_{C_{R}} \frac{e^{-\lambda\left(\mathrm{Re}^{i \theta}\right)^{\alpha}}}{\left(\operatorname{Re}^{i \theta}\right)^{\beta}} i \operatorname{Re}^{i \theta \theta} e^{t \mathrm{R} e^{i \theta}} d \theta \\
+ & \frac{1}{2 \pi i} \int_{R}^{\varepsilon} \frac{e^{-\lambda\left(r^{\alpha} e^{\alpha i(\pi-\delta)}\right)}}{r^{\beta} e^{i \beta(\pi-\delta)}} e^{i(\pi-\delta)} e^{r e^{i(\pi-\delta)}} d r
\end{aligned}
$$




$$
\begin{aligned}
& +\frac{1}{2 \pi i} \int_{C_{\varepsilon}} \frac{e^{-\lambda\left(\varepsilon^{\alpha} e^{i \alpha \theta}\right)}}{\varepsilon^{\beta} e^{i \beta \theta}} i \varepsilon e^{i \theta} e^{\varepsilon e^{i \theta}} d \theta \\
& +\frac{1}{2 \pi i} \int_{\varepsilon}^{R} \frac{e^{-\lambda\left(r^{\alpha} e^{-i \alpha(\pi-\delta)}\right)}}{r^{\beta} e^{-i \beta(\pi-\delta)}} e^{-i(\pi-\delta)} e^{r e^{-i(\pi-\delta)}} d r
\end{aligned}
$$

Now we show that the integrals along the $\operatorname{arcs} C_{R}, C_{R}^{\prime}$ tend to zero as $R \rightarrow \infty$,

$$
\left|I_{C_{R}}\right| \leq \frac{1}{|2 \pi i|} \int_{\theta_{1}}^{\pi-\delta} e^{-\lambda R^{\alpha} \cos \alpha \theta} R^{1-\beta} e^{t R \cos \theta} d \theta
$$

making a new change of variable $\alpha \theta=w$ one can rewrite the above integral as below,

$$
\left|I_{C_{R}}\right| \leq \frac{R^{1-\beta}}{2 \pi \alpha} \int_{\alpha \theta_{1}}^{\alpha(\pi-\delta)} e^{-\lambda R^{\alpha} \cos w} e^{t R \cos \frac{w}{\alpha}} d w
$$

again making a new change of variable $\varphi=\frac{\pi}{2}+w$ we have,

$$
\begin{aligned}
\left|I_{C_{R}}\right| & \leq \frac{R^{1-\beta}}{2 \pi \alpha} \int_{\alpha \theta_{1}+\frac{\pi}{2}}^{\alpha(\pi-\delta)+\frac{\pi}{2}} e^{-\lambda R^{\alpha} \sin \varphi} e^{t R \cos \left(\frac{2 \varphi-\pi}{2 \alpha}\right)} d \varphi \\
& \leq \frac{R^{1-\beta} e^{-\lambda R^{\alpha}}}{2 \pi \alpha} \int_{\alpha \theta_{1}+\frac{\pi}{2}}^{\alpha(\pi-\delta)+\frac{\pi}{2}} e^{t R \cos \left(\frac{2 \varphi-\pi}{2 \alpha}\right)} d \varphi .
\end{aligned}
$$

On the other hand, $\alpha \theta_{1}+\frac{\pi}{2}<\varphi<\alpha(\pi-\delta)+\frac{\pi}{2}$ therefore, $\theta_{1}<\frac{\varphi}{\alpha}-\frac{\pi}{2 \alpha}<\pi-\delta$. But we have $R \rightarrow \infty, \delta \rightarrow 0$ and consequently $\theta_{1} \rightarrow \frac{\pi}{2}$ so, $-1<\cos \left(\frac{2 \varphi+\pi}{2 \alpha}\right)<0$. Substituting in (1.4) and assuming $R \rightarrow \infty$ we get $\lim _{R \rightarrow \infty}\left|I_{C_{R}}\right|=0$. Similarly one can prove that $\lim _{R \rightarrow \infty}\left|I_{C_{R}^{\prime}}\right|=0$.

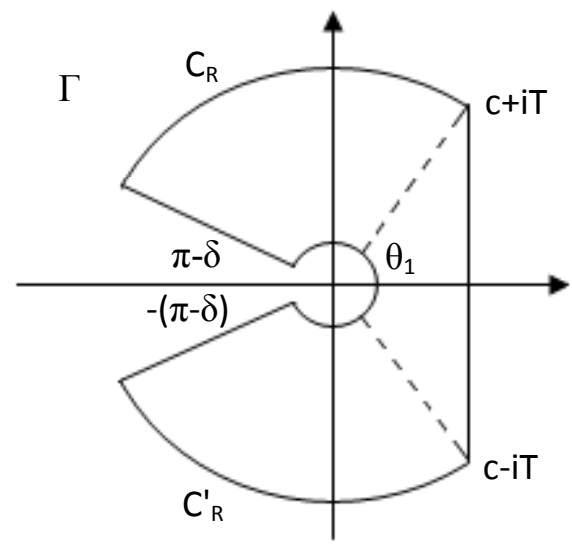

Figure 1.1
Now, let us evaluate the integral along the arc $C_{\varepsilon}$

$$
\left|I_{C_{\varepsilon}}\right| \leq \frac{1}{2 \pi} \int_{\pi-\delta}^{-(\pi-\delta)} e^{-\lambda\left(\varepsilon^{\alpha} \cos \alpha \theta\right)} \varepsilon^{1-\beta} d \theta
$$

which assuming $\varepsilon \rightarrow 0, \delta \rightarrow 0$ can be rewritten as

$$
\lim _{\varepsilon \rightarrow 0}\left|I_{C_{\varepsilon}}\right| \leq \lim _{\varepsilon \rightarrow 0} \frac{\varepsilon^{1-\beta}}{2 \pi} \int_{\pi}^{-\pi} d \theta=0
$$

substituting in (1.3) and letting $\delta \rightarrow 0$ we have

$$
\begin{aligned}
\frac{1}{2 \pi i} \int_{c-i \infty}^{c+i \infty} \frac{e^{-\lambda s^{\alpha}}}{s^{\beta}} e^{s t} d s= & \frac{1}{2 \pi i} \int_{0}^{\infty} \frac{e^{-\lambda\left(r^{\alpha} e^{i \alpha(\pi-\delta)}\right)}}{r^{\beta} e^{i \beta(\pi-\delta)}} e^{i(\pi-\delta)} e^{r e^{i(\pi-\delta)}} d r \\
& -\frac{1}{2 \pi i} \int_{0}^{\infty} \frac{e^{-\lambda\left(r^{\alpha} e^{-i \alpha(\pi-\delta)}\right)}}{r^{\beta} e^{-i \beta(\pi-\delta)}} e^{-i(\pi-\delta)} e^{r e^{-i(\pi-\delta)}} d r,
\end{aligned}
$$

or

$\frac{1}{2 \pi i} \int_{c-i \infty}^{c+i \infty} \frac{e^{-\lambda s^{\alpha}}}{s^{\beta}} d s=\frac{1}{2 \pi i}\left(\int_{0}^{\infty} e^{-r t} r^{-\beta} e^{-\lambda r^{\alpha} \cos \alpha \pi}\right.$

$\left.\left.\left\{2 i \cos \beta \pi \cdot \sin \left(\lambda r^{\alpha} \sin \alpha \pi\right)\right)+2 i \sin \beta \pi \cdot \cos \left(\lambda r^{\alpha} \sin \alpha \pi\right)\right) d r,\right)$ thus, the final solution is obtained as below

$$
\begin{aligned}
\frac{1}{2 \pi i} \int_{c-i \infty}^{c+i \infty} \frac{e^{-\lambda s^{\alpha}}}{s^{\beta}} e^{s t} d s= & \frac{1}{\pi}\left(\int_{0}^{\infty} e^{-r t} r^{-\beta} e^{-\lambda r^{\alpha} \cos \alpha \pi} \sin (\beta \pi\right. \\
& \left.\left.+\lambda r^{\alpha} \sin \alpha \pi\right)\right) d r .
\end{aligned}
$$

Problem 1.5 Evaluate the inverse Laplace transform of the function

$$
F(s)=\frac{\ln (s+\lambda)}{s+\mu} ; \quad 0<\mu<\lambda .
$$

Solution. By using the definition of the inverse Laplace transform one has

$$
L^{-1}\{F(s) ; t\}=\frac{1}{2 \pi i} \int_{c-i \infty}^{c+i \infty} e^{s t} \frac{\ln (s+\lambda)}{s+\mu} d s
$$

but the function $F(s)$ has a branch point at $s_{1}=-\lambda$ and a simple pole at $s_{2}=-\mu$. Now by integrating along the contour $\Gamma$ shown in figure 1.2 we have

$$
\begin{aligned}
\frac{1}{2 \pi i} \oint_{\Gamma} e^{s t} & \frac{\ln (s+\lambda)}{s+\mu} d s=\frac{1}{2 \pi i} \int_{\gamma-i T}^{\gamma+i T} e^{s t} \frac{\ln (s+\lambda)}{s+\mu} d s \\
& +\frac{1}{2 \pi i} \int_{C_{R}} e^{s t} \frac{\ln (s+\lambda)}{s+\mu} d s+\frac{1}{2 \pi i} \int_{C_{R}^{\prime}} e^{s t} \frac{\ln (s+\lambda)}{s+\mu} d s
\end{aligned}
$$




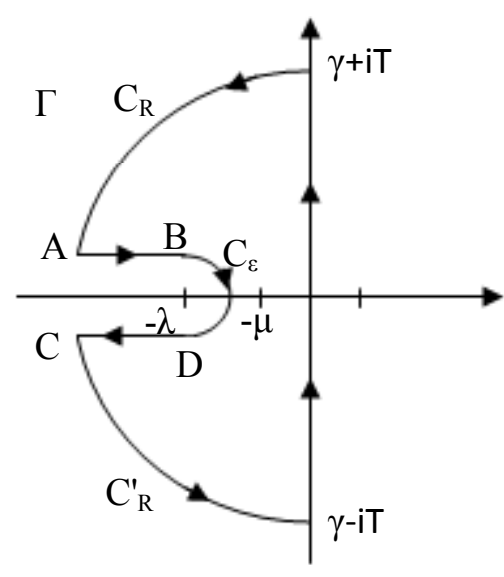

Figure 1.2

$$
\begin{aligned}
& +\frac{1}{2 \pi i} \int_{C_{\varepsilon}} e^{s t} \frac{\ln (s+\lambda)}{s+\mu} d s+\frac{1}{2 \pi i} \int_{A B} e^{s t} \frac{\ln (s+\lambda)}{s+\mu} d s \\
& +\frac{1}{2 \pi i} \int_{C D} e^{s t} \frac{\ln (s+\lambda)}{s+\mu} d s=e^{-\mu t} \ln (\lambda-\mu),
\end{aligned}
$$

in which $e^{-\mu t} \ln (\lambda-\mu)=\operatorname{Re} s\left\{e^{s t} \frac{\ln (s+\lambda)}{s+\mu} ; s=-\mu\right\}$. One can show that the integration along the $\operatorname{arcs} C_{R}, C_{R}^{\prime}$ and $C_{\varepsilon}$ tends to zero. Therefore

$$
\begin{array}{r}
\frac{1}{2 \pi i} \int_{\gamma-i T}^{\gamma+i T} e^{s t} \frac{\ln (s+\lambda)}{s+\mu} d s=-\frac{1}{2 \pi i} \int_{A B} e^{s t} \frac{\ln (s+\lambda)}{s+\mu} d s \\
-\frac{1}{2 \pi i} \int_{C D} e^{s t} \frac{\ln (s+\lambda)}{s+\mu} d s-e^{-\mu t} \ln (\lambda-\mu) .
\end{array}
$$

It suffices to evaluate the integrals along the segments $A B, C D$. We have

$$
\begin{aligned}
I & =\frac{1}{2 \pi i} \int_{A B} \frac{\ln (s+\lambda)}{s+\mu} e^{s t} d s+\frac{1}{2 \pi i} \int_{C D} \frac{\ln (s+\lambda)}{s+\mu} e^{s t} d s \\
& =\frac{1}{2 \pi i} \int_{-\infty}^{-1-\varepsilon} \frac{\ln |x+\lambda|+i \pi}{x+\mu} e^{x t} d x+\frac{1}{2 \pi i} \int_{-1-\varepsilon}^{-\infty} \frac{\ln |x+\lambda|-i \pi}{x+\mu} e^{x t} d x,
\end{aligned}
$$

making a new change of variable $u=-x$, one can rewrite the above integration as below

$$
I=\frac{1}{2 \pi i} \int_{1+\varepsilon}^{\infty} \frac{\ln (x-\lambda)+i \pi}{\mu-x} e^{-x t} d x-\frac{1}{2 \pi i} \int_{1+\varepsilon}^{\infty} \frac{\ln (x-\lambda)-i \pi}{\mu-x} e^{-x t} d x,
$$

if $\varepsilon$ tends to zero we have

$$
\begin{aligned}
I & =-\frac{1}{2 \pi i} \int_{1}^{\infty} \frac{2 \pi i}{x-\mu} e^{-x t} d x=e^{-\mu t} \int_{1-\mu}^{\infty} \frac{e^{-u t}}{u} d u \\
& =e^{-\mu t} \int_{(1-\mu) t}^{\infty} \frac{e^{-w}}{w} d w=-e^{-\mu t} E_{i}((\mu-1) t)
\end{aligned}
$$

substituting in (1.6) we obtain

$$
\begin{aligned}
\frac{1}{2 \pi i} \int_{\gamma-i T}^{\gamma+i T} e^{s t} \frac{\ln (s+\lambda)}{s+\mu} d s & =e^{-\mu t} E_{i}((\mu-1) t)-e^{-\mu t} \ln (\lambda-\mu) \\
& =-\left(e^{-\mu t} E_{1}((1-\mu) t)+\ln (\lambda-\mu)\right)
\end{aligned}
$$

LEMMA 1.6 (TitchmARsh) Let $F(p)$ be an analytic function having no singularities in the cut plane $C \backslash R$. Assume that $\overline{F(p)}=F(\bar{p})$ and the limiting values

$$
F^{ \pm}(t)=\lim _{\phi \rightarrow \pi^{-}} F\left(t e^{ \pm i \phi}\right), \quad F^{+}(t)=\overline{F^{-}(t)}
$$

exist for almost all

(i) $F(p)=o(1)$ for $|p| \rightarrow \infty$ and $F(p)=o\left(|p|^{-1}\right)$ for $|p| \rightarrow 0$, uniformly in any sector $|\arg p|<\pi-\eta, \pi>\eta>0$;

(ii) There exists $\varepsilon>0$ such that for every $\pi-\varepsilon<\phi \leq \pi$,

$$
\frac{F\left(r e^{ \pm i \phi}\right)}{1+r} \in L^{1}\left(R_{+}\right), \quad\left|F\left(r e^{ \pm i \phi}\right)\right| \leq a(r)
$$

where $a(r)$ does not depend on $\phi$ and $a(r) e^{-\delta r} \in L^{1}\left(R_{+}\right)$ for any $\delta>0$. Then, in the notation of the problem,

$$
f(t)=L^{-1}[F(s)]=\frac{1}{\pi} \int_{0}^{\infty} \operatorname{Im}\left[F^{-}(\eta)\right] e^{-t \eta} d \eta .
$$

Proof. See ${ }^{13,16}$.

Problem 1.7 Find inverse Laplace transform of the function discussed in problem 1.4

$$
F(s)=\frac{e^{-\lambda s^{\alpha}}}{s^{\beta}} ; \quad 0<\alpha<1, \quad 0 \leq \beta<1 .
$$

by using Titchmarsh formula in lemma1.6.

Solution. By using lemma 1.6 we have

$$
L^{-1}\{F(s) ; t\}=f(t)=\frac{1}{\pi} \int_{0}^{\infty} \operatorname{Im}\left[\lim _{\phi \rightarrow \pi^{-}} F\left(\eta e^{-i \phi}\right)\right] e^{-t \eta} d \eta,
$$

substituting the function $F(s)$ in the above formula we get

$$
L^{-1}\left\{\frac{e^{-\lambda s^{\alpha}}}{s^{\beta}} ; t\right\}=\frac{1}{\pi} \int_{0}^{\infty} \operatorname{Im}\left(\frac{e^{-\lambda\left(\eta e^{-i \pi}\right)^{\alpha}}}{\left(\eta e^{-i \pi}\right)^{\beta}}\right) e^{-t \eta} d \eta
$$

therefore, the result is obtained as

$$
L^{-1}\left\{\frac{e^{-\lambda s^{\alpha}}}{s^{\beta}} ; t\right\}=\frac{1}{\pi} \int_{0}^{\infty} \frac{e^{-\lambda \eta^{\alpha} \cos \alpha \pi}}{\eta^{\beta}} \sin \left(\pi \beta+\lambda \eta^{\alpha} \sin \pi \alpha\right) e^{-t \eta} d \eta,
$$


one can observe that the result which obtained here is exactly the same as relationship (1.5).

Difinition 1.8 Finite Fourier sine and cosine transforms are defined as

$$
\begin{aligned}
& F_{s}\{f(x) ; n\}=F_{s}(n)=\int_{0}^{L} f(x) \sin \frac{n \pi x}{L} d x, \\
& F_{c}\{f(x) ; n\}=F_{c}(n)=\int_{0}^{L} f(x) \cos \frac{n \pi x}{L} d x,
\end{aligned}
$$

while their inverse are as below

$$
\begin{aligned}
& F_{s}^{-1}\left\{F_{s}(n) ; x\right\}=f(x)=\frac{2}{L} \sum_{n=1}^{\infty} F_{s}(n) \sin \frac{n \pi x}{L}, \\
& F_{c}^{-1}\left\{F_{c}(n) ; x\right\}=f(x)=\frac{1}{L} F_{c}(0)+\frac{2}{L} \sum_{n=1}^{\infty} F_{c}(n) \cos \frac{n \pi x}{L} .
\end{aligned}
$$

Difinition 1.9 Laguerre differential equation is defined as

$$
x y^{\prime \prime}+(1-x) y^{\prime}+n y=0 ; \quad y(0)=n !,
$$

which can be solved by using Laplace transform .Let us assume that

$$
L\{y(x)\}=L\left\{L_{n}(x)\right\}=F(s),
$$

taking Laplace transform of Laguerre differential equation we obtain

$$
F(s)=\frac{1}{s}\left(1-\frac{1}{s}\right)^{n} \ddot{=} L_{n}(x)
$$

LEMмA 1.10: The following integral relations hold true.

$$
\begin{aligned}
& \int_{0}^{1} x^{\lambda-1} \operatorname{bei}(2 \sqrt{|\ln x|}) d x=\lambda^{-1} \sin \lambda^{-1}, 0<x<1 \\
& \int_{0}^{1} \frac{b e i(2 \sqrt{|\ln x|}) d x}{2 \sqrt{x}}=\sin 2
\end{aligned}
$$

Proof: Let us define the following integral

$$
I(\xi)=\int_{0}^{1} x^{\lambda-1} \operatorname{bei}(2 \sqrt{\ln x \mid} \xi) d x
$$

Now, we calculate Laplace transform of the above relation to obtain

$$
\begin{aligned}
L\{I(\xi), s\} & =\int_{0}^{+\infty} e^{-s \xi}\left\{\int_{0}^{1} x^{\lambda-1} b e i(2 \sqrt{|\ln x|} \xi) d x\right\} d \xi \\
& =\int_{0}^{1} x^{\lambda-1}\left\{s^{-1} \sin \left(|\ln x| s^{-1}\right)\right\} d x
\end{aligned}
$$

In the last integral, let us introduce the new variable, $w=-\ln x$

By setting the above change of variable in the last integral, we get

$$
L\{I(\xi), s\}=s^{-1} \int_{0}^{+\infty} e^{-\lambda w} \sin \left(s^{-1} w\right) d w=\frac{1}{\lambda^{2} s^{2}+1}
$$

Upon inversion, one gets the following relationship

$$
I(\xi)=L^{-1}\left\{\frac{\lambda^{-1}}{\lambda\left(s^{2}+\lambda^{-2}\right)}\right\}=\frac{1}{\lambda} \sin \frac{\xi}{\lambda}
$$

At this point, by setting $\xi=1$, one gets

$$
\int_{0}^{1} x^{\lambda-1} \operatorname{bei}(2 \sqrt{|\ln x|}) d x=\lambda^{-1} \sin \lambda^{-1}
$$

For special case $\lambda=0.5$, we obtain

$$
\int_{0}^{1} \frac{b e i(2 \sqrt{|\ln x|}) d x}{2 \sqrt{x}}=2 \sin 2
$$

\section{Two Dimensional Laplace Transform}

Definition 2.1 Two dimensional Laplace transform of the function $f(x, y)$ is defined as

$$
F(p, q)=\int_{0}^{+\infty} \int_{0}^{+\infty} e^{-p x-q y} f(x, y) d x d y
$$

while its inverse is given by

$$
f(x, y)=\frac{1}{2 \pi i} \int_{c-i \infty \infty}^{c+i \infty} \int_{c^{\prime}-i \infty}^{c^{\prime}+\infty} F(p, q) e^{p x+q y} d p d q,
$$

where $F(p, q)$ is analytic in the region $\operatorname{Re} p>c, \operatorname{Re} q>c^{\prime 3-6}$.

Lemma 2.2 Let us assume that $L\{f(t)\}=F(s)$, then the following relationship holds true

$$
L_{2}^{(p, q)}\{f(a x+b y)\}=\frac{1}{b p-a q}\left\{F\left(\frac{q}{b}\right)-F\left(\frac{p}{a}\right)\right\} .
$$

Proof. By definition of two dimensional Laplace transform we have

$$
L_{2}^{(p, q)}\{f(a x+b y)\}=\int_{0}^{+\infty} \int_{0}^{+\infty} e^{-p x-q y} f(a x+b y) d x d y,
$$

now, making a change of variables $a x+b y=u$, in the inner integral we obtain 


$$
L_{2}^{(p, q)}\{f(a x+b y)\}=\frac{1}{a} \int_{0}^{+\infty} e^{-q y} \int_{b y}^{+\infty} e^{-p\left(\frac{u-b y}{a}\right)} f(u) d u d y,
$$

changing the order of integrals to get

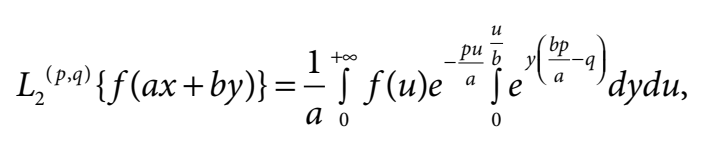

consequently, the final result is obtained as below

$$
L_{2}^{(p, q)}\{f(a x+b y)\}=\frac{1}{b p-a q}\left\{F\left(\frac{q}{b}\right)-F\left(\frac{p}{a}\right)\right\} .
$$

EXAMPLE 2.3 Evaluate

$$
L_{2}^{(p, q)}\left\{\ln \left(\frac{9 x^{2}+12 x y+4 y^{2}}{4 x y}\right)\right\} .
$$

Solution. By definition we have

$$
\begin{aligned}
L_{2}^{(p, q)}\left\{\ln \left(\frac{9 x^{2}+12 x y+4 y^{2}}{4 x y}\right)\right\}= & L_{2}^{(p, q)}\left\{\ln (3 x+2 y)^{2}\right\} \\
& -L_{2}^{(p, q)}\{\ln (4 x y)\}
\end{aligned}
$$

from lemma 2.2 we can write

$$
\begin{aligned}
L_{2}^{(p, q)}\left\{\ln (3 x+2 y)^{2}\right\}= & \frac{2}{2 p-3 q}\left\{\frac{3}{p} \ln \left(\frac{p}{3}\right)-\frac{2}{q} \ln \left(\frac{q}{2}\right)\right. \\
& \left.+\gamma\left(\frac{3}{p}-\frac{2}{q}\right)\right\},
\end{aligned}
$$

on the other hand, by using definition we know that

$$
L_{2}^{(p, q)}\{\ln (4 x y)\}=-\frac{1}{p q}\left(\ln \left(\frac{p}{4}\right)+\ln q+2 \gamma\right),
$$

in which $\gamma=0.5772 \ldots$ is the Euler constant. Therefore, the final result will be

$$
\begin{aligned}
& L_{2}^{(p, q)}\left\{\ln \left(\frac{9 x^{2}+12 x y+4 y^{2}}{4 x y}\right)\right\} \\
& \quad=\frac{2}{2 p-3 q}\left\{\frac{3}{p} \ln \left(\frac{p}{3}\right)-\frac{2}{q} \ln \left(\frac{q}{2}\right)\right\}+\frac{1}{p q}\left(\ln \left(\frac{p}{4}\right)+\ln q\right) .
\end{aligned}
$$

Proposition 2.4 The following relationship holds true

$$
\int_{0}^{\infty} \int_{0}^{\infty} e^{-\left(x^{2}+2 x y \cos \theta+y^{2}\right)} d x d y=\frac{\pi}{\sin \theta}
$$

Proof. Consider the change of variables as below by using matrix of spinning $45^{\circ}$ around the origin

$$
\left\{\begin{array}{l}
\xi=\frac{1}{\sqrt{2}} x-\frac{1}{\sqrt{2}} y \\
\eta=\frac{1}{\sqrt{2}} x+\frac{1}{\sqrt{2}} y
\end{array}\right.
$$

substituting in (2.3) one gets

$$
x^{2}+2 x y \cos \theta+y^{2}=\xi^{2}+\eta^{2}+\left(\xi^{2}-\eta^{2}\right) \cos \theta,|J|=1,
$$

in which $\mathrm{J}$ is the Jacobian determinant. Now, from definition 2.1 we have

$$
\begin{aligned}
& L_{2}\left\{\exp \left[-\left(x^{2}+2 x y \cos \theta+y^{2}\right)\right]\right\} \\
& =\int_{0}^{\infty} \int_{0}^{\infty} \exp \left(-\xi^{2}-\eta^{2}-\left(\eta^{2}-\xi^{2}\right) \cos \theta\right) e^{\frac{p(\xi+\eta)}{\sqrt{2}}} e^{\frac{q(\eta-\xi)}{\sqrt{2}}} d \xi d \eta,
\end{aligned}
$$

or one can rewrite the above equation as below

$$
\begin{aligned}
& \int_{0}^{\infty} e^{\xi^{2}(\cos \theta-1)+\frac{\xi(p-q)}{\sqrt{2}}} d \xi \times \int_{0}^{\infty} e^{-\eta^{2}(1+\cos \theta)+\frac{\eta(p+q)}{\sqrt{2}}} d \eta \\
& =\frac{\pi}{\sin \theta} \exp \left(\frac{p^{2}-q^{2}}{16 \sin ^{2} \theta}\right) \operatorname{erfc}\left(\frac{p+q}{2 \sqrt{2}(1-\cos \theta)^{\frac{1}{2}}}\right) \\
& \quad \times \operatorname{erfc}\left(\frac{p+q}{2 \sqrt{2}(1+\cos \theta)^{\frac{1}{2}}}\right) .
\end{aligned}
$$

Now, setting $p=q=0$ to get the desired relationship

$$
\int_{0}^{\infty} \int_{0}^{\infty} e^{-\left(x^{2}+2 x y \cos \theta+y^{2}\right)} d x d y=\frac{\pi}{\sin \theta}
$$

TheOrem 2.5 Consider the function

$$
h(x, y)=\int_{\max (x, y)}^{+\infty} f(u-x) g(u-y) k(u) d u,
$$

the following relationship holds true

$$
L_{2}^{(p, q)}\{h(x, y)\}=\int_{0}^{\infty} e^{-u(p+q)} k(u)\left\{\int_{0}^{u} \int_{0}^{u} e^{p x+q y} f(x) g(y) d x d y\right\} d u .
$$

Proof. By definition of two dimensional Laplace transform, one has

$$
L_{2}^{(p, q)}\{h(x, y)\}=\int_{0}^{\infty} \int_{0}^{\infty} e^{-p x-q y} \int_{\max (x, y)}^{+\infty} f(u-x) g(u-y) k(u) d u,
$$


now, we consider two cases for $h(x, y): y>x$ and $y<x$ and integrate over these regions and add them together to obtain

$$
\begin{aligned}
L_{2}^{(p, q)}\{h(x, y)\} & =\int_{0}^{\infty} e^{-q y} \int_{0}^{y} e^{-p x} \int_{y}^{+\infty} f(u-x) g(u-y) k(u) d u d x d y \\
& +\int_{0}^{+\infty} e^{-p x} \int_{0}^{x} e^{-q y} \int_{x}^{+\infty} f(u-x) g(u-y) k(u) d u d y d x,
\end{aligned}
$$

changing the order of the inner integrals we have

$$
\begin{aligned}
=\int_{0}^{\infty} e^{-q y} \int_{y}^{\infty} g(u-y) k(u) \int_{0}^{y} e^{-p x} f(u-x) d x d u d y \\
+\int_{0}^{\infty} e^{-p x} \int_{x}^{\infty} f(u-x) k(u) \int_{0}^{x} e^{-q y} g(u-y) d y d u d x,
\end{aligned}
$$

by a change of variables $u-x=\eta$ in the first triple integral and $u-y=\xi$ in the second triple integral, one gets

$$
\begin{aligned}
= & \int_{0}^{\infty} e^{-q y} \int_{y}^{\infty} e^{-p u} g(u-y) k(u) \int_{u-y}^{u} e^{p \eta} f(\eta) d \eta d u d y \\
& +\int_{0}^{\infty} e^{-p x} \int_{x}^{\infty} e^{-q u} f(u-x) k(u) \int_{u-x}^{u} e^{q \xi} g(\xi) d \xi d u d x,
\end{aligned}
$$

now we change the order of outer integrals in both terms of the above equation, to get

$$
\begin{aligned}
=\int_{0}^{\infty} e^{-p u} k(u) \int_{0}^{u} e^{-q y} g(u-y) \int_{u-y}^{u} e^{p \eta} f(\eta) d \eta d y d u \\
+\int_{0}^{\infty} e^{-q u} k(u) \int_{0}^{u} e^{-p x} f(u-x) \int_{u-x}^{u} e^{q \xi} g(\xi) d \xi d x d u,
\end{aligned}
$$

again by a change of new variables $u-x=\eta$ in the second triple integral and $u-y=\xi$ in the first triple integral we have

$$
\begin{aligned}
=\int_{0}^{\infty} e^{-u(p+q)} & k(u) \int_{0}^{u} e^{q \xi} g(\xi) \int_{\xi}^{u} e^{p \eta} f(\eta) d \eta d \xi d u \\
& +\int_{0}^{\infty} e^{-u(p+q)} k(u) \int_{0}^{u} e^{p \eta} f(\eta) \int_{\eta}^{u} e^{q \xi} g(\xi) d \xi d \eta d u,
\end{aligned}
$$

it means that

$$
\begin{aligned}
L_{2}^{(p, q)}\{h(x, y)\}= & \int_{0}^{\infty} e^{-u(p+q)} k(u)\left(\int_{0}^{u} e^{q \xi} g(\xi) \int_{\xi}^{u} e^{p \eta} f(\eta) d \eta d \xi\right. \\
& \left.+\int_{0}^{u} e^{p \eta} f(\eta) \int_{\eta}^{u} e^{q \xi} g(\xi) d \xi d \eta\right) d u,
\end{aligned}
$$

and consequently

$$
L_{2}^{(p, q)}\{h(x, y)\}=\int_{0}^{\infty} e^{-u(p+q)} k(u)\left\{\int_{0}^{u} \int_{0}^{u} e^{p x+q y} f(x) g(y) d x d y\right\} d u .
$$

LEMMA 2.6 The following relationship holds true

$$
\begin{aligned}
& L_{2}^{(p, q)}\left\{K_{0}(x+y) ; x \rightarrow p, y \rightarrow q\right\} \\
& =\frac{1}{q-p}\left(\frac{\cos ^{-1} p}{\sqrt{1-p^{2}}}-\frac{\cos ^{-1} q}{\sqrt{1-q^{2}}}\right) .
\end{aligned}
$$

Proof. By using the following integral representation we have

$$
K_{0}(x+y)=\int_{0}^{\infty} \cos ((x+y) \sinh \theta) d \theta .
$$

Now, from definition of two dimensional Laplace transform one has

$$
L_{2}^{(p, q)}\left\{K_{0}(x+y)\right\}=\int_{0}^{\infty} \int_{0}^{\infty} e^{-(p x+q y)}\left(\int_{0}^{\infty} \cos ((x+y) \sinh \theta) d \theta\right) d x d y,
$$

by using the following elementary relationship

$$
\begin{aligned}
\cos (x \sinh \theta+y \sinh \theta)= & \cos (x \sinh \theta) \cos (y \sinh \theta) \\
& -\sin (x \sinh \theta) \sin (y \sinh \theta)
\end{aligned}
$$

and changing the order of integrals, we can write

$$
\begin{aligned}
L_{2}^{(p, q)}\left\{K_{0}(x+y)\right\} & =\int_{0}^{\infty} \frac{p q-\sinh ^{2} \theta}{\left(p^{2}+\sinh ^{2} \theta\right)\left(q^{2}+\sinh ^{2} \theta\right)} d \theta \\
& =\int_{0}^{\infty}\left(\frac{p}{p^{2}+\sinh ^{2} \theta}-\frac{q}{q^{2}+\sinh ^{2} \theta}\right) \frac{d \theta}{q-p} .
\end{aligned}
$$

By manipulating similarly to what we have done in lemma 2.2, one gets finally

$L_{2}^{(p, q)}\left\{K_{0}(x+y) ; x \rightarrow p, y \rightarrow q\right\}=\frac{1}{q-p}\left(\frac{\cos ^{-1} p}{\sqrt{1-p^{2}}}-\frac{\cos ^{-1} q}{\sqrt{1-q^{2}}}\right)$.

Corollary 2.7 The following relationship holds true

$$
L_{2}^{(p, q)}\left\{K_{0}|x-y|\right\}=\frac{1}{p+q}\left(\frac{\arccos p}{\sqrt{1-p^{2}}}+\frac{\arccos q}{\sqrt{1-q^{2}}}\right) .
$$

Proof. By using the following integral representation for $K_{0}(x)$

$$
K_{0}(x)=\int_{0}^{\infty} \cos (x \sinh \theta) d \theta
$$


we have

$$
K_{0}|x-y|=\int_{0}^{\infty} \cos (|x-y| \sinh \theta) d \theta .
$$

Because of the symmetry property of the cosine function we can write

$$
K_{0}|x-y|=\int_{0}^{\infty} \cos ((x-y) \sinh \theta) d \theta .
$$

Now, from definition of two dimensional Laplace transform and lemma 2.5 we have

$$
\begin{aligned}
& L_{2}^{(p, q)}\left\{K_{0}|x-y|\right\} \\
& \quad=\int_{0}^{\infty} \int_{0}^{\infty} e^{-(p x+q y)}\left(\int_{0}^{\infty} \cos ((x-y) \sinh \theta) d \theta\right) d x d y \\
& =\frac{1}{p+q}\left(\frac{\arccos p}{\sqrt{1-p^{2}}}+\frac{\arccos q}{\sqrt{1-q^{2}}}\right) .
\end{aligned}
$$

COROLlarY 2.8 The following relationship holds true

$$
\begin{aligned}
& L_{2}^{p, q}\left\{K_{0}\left(\sqrt{x^{2}+2 x y \cos \theta+y^{2}}\right)\right\} \\
& =\frac{\pi}{2 \sin ^{2} \theta} \int_{0}^{\infty} e^{t\left(\frac{p^{2}+q^{2}-2 p q \cos \theta}{\sin ^{2} \theta}-1\right)} \operatorname{erfc}\left(\frac{(p-q) \sqrt{t}}{2 \sin \frac{\theta}{2}}\right) \operatorname{erfc}\left(\frac{(p+q) \sqrt{t}}{2 \cos \frac{\theta}{2}}\right) d t .
\end{aligned}
$$

Proof. By using the following integral representation for modified Bessel function of the second kind or the Mac - Donald function of order zero

$$
K_{0}(z)=\frac{1}{2} \int_{0}^{\infty} \exp \left(-t-\frac{z^{2}}{4 t}\right) \frac{d t}{t}, \quad z>0,
$$

and substituting $z=\sqrt{x^{2}+2 x y \cos \theta+y^{2}}$ we have

$$
K_{0}\left(\sqrt{x^{2}+2 x y \cos \theta+y^{2}}\right)=\frac{1}{2} \int_{0}^{\infty} e^{-t-\frac{x^{2}+2 x y \cos \theta+y^{2}}{4 t}} \frac{d t}{t},
$$

now, by definition of Laplace transform we get

$$
\begin{aligned}
& L_{2}^{p, q}\left\{K_{0}\left(\sqrt{x^{2}+2 x y \cos \theta+y^{2}}\right)\right\} \\
& \quad=\frac{1}{2} \int_{0}^{\infty} \int_{0}^{\infty} e^{-p x-q y}\left\{\int_{0}^{\infty} e^{-t-\frac{x^{2}+2 x y \cos \theta+y^{2}}{4 t}} \frac{d t}{t}\right\} d x d y,
\end{aligned}
$$

it suffices to change the order of integrals and use the change of variables

$$
\left\{\begin{array}{l}
\eta=\frac{x}{\sqrt{2}}-\frac{y}{\sqrt{2}} \\
\xi=\frac{x}{\sqrt{2}}+\frac{y}{\sqrt{2}}
\end{array},\right.
$$

to get the following relationship after some manipulations

$$
\begin{aligned}
& L_{2}^{p, q}\left\{K_{0}\left(\sqrt{x^{2}+2 x y \cos \theta+y^{2}}\right)\right\} \\
& =\frac{1}{2} \int_{0}^{\infty} \frac{e^{-t}}{t}\left(\int_{0}^{\infty} e^{\frac{\eta}{\sqrt{2}}(q-p)-\eta^{2} \frac{1-\cos \theta}{4 t}} d \eta\right)\left(\int_{0}^{\infty} e^{-\frac{\xi}{\sqrt{2}}(q+p)-\xi^{2} \frac{1+\cos \theta}{4 t}} d \xi\right) d t \text {, }
\end{aligned}
$$

now, considering two inner integrals as Laplace transform of functions the final result will be

$$
\begin{aligned}
& L_{2}^{p, q}\left\{K_{0}\left(\sqrt{x^{2}+2 x y \cos \theta+y^{2}}\right)\right\} \\
& =\frac{\pi}{2 \sin ^{2} \theta} \int_{0}^{\infty} e^{t\left(\frac{p^{2}+q^{2}-2 p q \cos \theta}{\sin ^{2} \theta}-1\right)} \operatorname{erfc}\left(\frac{(p-q) \sqrt{t}}{2 \sin \frac{\theta}{2}}\right) \operatorname{erfc}\left(\frac{(p+q) \sqrt{t}}{2 \cos \frac{\theta}{2}}\right) d t .
\end{aligned}
$$

LEMMA 2.9 (Evaluation of convergent series by means of two dimensional Laplace transform) Show that

$$
S=\sum_{n=0}^{\infty} L_{n}(\lambda x) L_{n}(\mu y)=\frac{e^{\mu y}}{\lambda} \delta\left(x-\frac{\mu}{\lambda} y\right) .
$$

Proof. From definition 1.9 we know that ${ }^{8}$

$$
L\left\{L_{n}(x)\right\}=\frac{1}{p}\left(1-\frac{1}{p}\right)^{n},
$$

and consequently

$$
L\left\{L_{n}(\lambda x)\right\}=\frac{1}{p}\left(1-\frac{\lambda}{p}\right)^{n},
$$

(Since $\left.L\left\{f\left(\frac{x}{a}\right)\right\}=a F(a p)\right)$. Now, taking two dimensional Laplace transform of series $S$ to get

$$
\begin{aligned}
L_{2}^{(p, q)}\{S\} & =\sum_{n=0}^{\infty}\left\{\frac{1}{p}\left(1-\frac{\lambda}{p}\right)^{n} \times \frac{1}{q}\left(1-\frac{\mu}{q}\right)^{n}\right\} \\
& =\sum_{n=0}^{\infty} \frac{1}{p q}\left(1-\frac{\lambda}{p}\right)^{n}\left(1-\frac{\mu}{q}\right)^{n},
\end{aligned}
$$

and this is a geometric series which converges to

$$
\frac{1}{p q} \times \frac{1}{1-\left(1-\frac{\lambda}{p}\right)\left(1-\frac{\mu}{q}\right)}=\frac{1}{\lambda q+\mu p-\lambda \mu} .
$$

Then taking the inverse two dimensional Laplace transform leads to

$$
\begin{aligned}
S & =L_{2}^{-1}\left\{\frac{1}{\lambda q+\mu p-\lambda \mu}\right\} \\
& =\frac{1}{2 \pi i} \int_{c-i \infty}^{c+i \infty} e^{q y}\left(\frac{1}{2 \pi i} \int_{c^{\prime}-i \infty}^{c^{\prime}+i \infty} \frac{e^{p x}}{\lambda q+\mu p-\lambda \mu} d p\right) d q
\end{aligned}
$$




$$
\begin{aligned}
& =\frac{1}{\mu} \times \frac{1}{2 \pi i} \int_{c-i \infty}^{c+i \infty} \exp \left(-\frac{\lambda q-\lambda \mu}{\mu} x\right) e^{q y} d q \\
& =\frac{e^{\lambda x}}{\mu} \delta\left(y-\frac{\lambda x}{\mu}\right),
\end{aligned}
$$

or by replacing $x$ by $y$ and $y$ by $x$ we get

$$
S=\frac{e^{\mu y}}{\lambda} \delta\left(x-\frac{\mu}{\lambda} y\right)
$$

LEMм 2.10 The following relationship holds true

$$
I=\int_{0}^{\infty} \frac{\operatorname{ber}(\sqrt[4]{\xi}) K_{0}(2 \sqrt{\xi}) d \xi}{\sqrt{\xi}}=\frac{\pi}{2} J_{0}\left(\frac{1}{8}\right)
$$

Proof. By making a change of variables $\sqrt{\xi}=u$ we have

$$
I=2 \int_{0}^{\infty} \operatorname{ber}(\sqrt{u}) K_{0}(2 u) d u,
$$

now introducing the following integral by using a new variable $t$

$$
I(t)=2 \int_{0}^{\infty} \operatorname{ber}(\sqrt{u t}) K_{0}(2 u) d u,
$$

it suffices to take Laplace transform of $I(t)$ with respect to $t$ to obtain

$$
\begin{aligned}
L\{I(t) ; t \rightarrow s\} & =\frac{2}{s} \int_{0}^{\infty} \cos \frac{u}{4 s} K_{0}(2 u) d u \\
& =\frac{2}{s} \frac{\pi}{2 \sqrt{\frac{1}{16 s^{2}}+4}}=\frac{\pi}{2 \sqrt{s^{2}+\frac{1}{64}}},
\end{aligned}
$$

consequently, the final result will be obtained by taking inverse Laplace transform and letting $t=1$ as below

$$
I=I(t=1)=L^{-1}\left\{\frac{\pi}{2 \sqrt{s^{2}+\frac{1}{64}}} ; s \rightarrow t=1\right\}=\frac{\pi}{2} J_{0}\left(\frac{1}{8}\right) .
$$

\section{Mathieu Series}

Thefollowing infinite series

$$
S(\lambda)=\sum_{n=1}^{\infty} \frac{2 n}{\left(n^{2}+\lambda^{2}\right)^{2}}, \quad\left(\lambda \in R^{+}\right)
$$

is named after Émile Leonard Mathieu (1835-1890), who is investigated in his work on elasticity of solid bodies. An alternating version of Mathieu series (4.1) is in the form

$$
\tilde{S}(\lambda)=\sum_{n=1}^{\infty}(-1)^{n-1} \frac{2 n}{\left(n^{2}+\lambda^{2}\right)^{2}}, \quad\left(\lambda \in R^{+}\right)
$$

our purpose is to obtain new integral representations for the above series, it is obvious that the series are convergent $\left(\operatorname{see}^{11}\right)$.

LEMMA 3.1 The following relationship holds true for the Mathieu series

$$
\begin{aligned}
S & =\sum_{n=1}^{\infty} \frac{2 n}{\left(n^{2}+\lambda^{2}\right)^{2}} \\
& =\frac{1}{2 \lambda\left(1-e^{-2 \pi \lambda}\right)^{2}} \int_{0}^{2 \pi} e^{-\lambda y}\left((2 \pi-y) e^{-2 \pi \lambda}+y\right) \cot \frac{y}{2} d y .
\end{aligned}
$$

Proof. By definition of two dimensional Laplace transform we have

$$
\begin{aligned}
F(p, q) & =L_{2}^{(p, q)}\{\sin \lambda(x+y)\} \\
& =\int_{0}^{\infty} e^{-q y}\left(\int_{0}^{\infty} \sin \lambda(x+y) e^{-p x} d x\right) d y \\
& =\int_{0}^{\infty} e^{-q y}\left\{\frac{p \sin \lambda y+\lambda \cos \lambda y}{p^{2}+\lambda^{2}}\right\} d y \\
& =\frac{\lambda(p+q)}{\left(q^{2}+\lambda^{2}\right)\left(p^{2}+\lambda^{2}\right)} .
\end{aligned}
$$

We know that $\left(\right.$ see $\left.^{2}\right)$

$$
\sum_{n=1}^{\infty} F(n, n)=\int_{0}^{\infty} \frac{1}{e^{w}-1}\left(\int_{0}^{w} \sin \lambda w d y\right) d w
$$

it means that

$$
\sum_{n=1}^{\infty} \frac{2 n}{\left(n^{2}+\lambda^{2}\right)^{2}}=\frac{1}{\lambda} \int_{0}^{\infty} \frac{w \sin \lambda w}{e^{w}-1} d w
$$

Let us evaluate the following integral

$$
I(\lambda)=\int_{0}^{\infty} \frac{\cos \lambda w}{e^{w}-1} d w
$$

and then differentiate it with respect to $\lambda$ to get the desired result. For this purpose, let us consider the complex function

$$
f(z)=\frac{e^{i \lambda z}}{e^{z}-1}
$$

with simple poles at points $z=0,2 \pi i$. Consider the following integral along the path which is shown in figure 3.1 


$$
\frac{1}{2 \pi i} \oint_{\Gamma} f(z) d z=0
$$

Moving along the path $\Gamma$ counterclockwise we get

$$
\begin{aligned}
\int_{\varepsilon}^{R} \frac{e^{i \lambda w}}{e^{w}-1} d w & +\int_{0}^{2 \pi} \frac{e^{i \lambda(R+i y)}}{e^{R+i y}-1} i d y+\int_{R}^{\varepsilon} \frac{e^{i \lambda(w+2 \pi i)}}{e^{w+2 \pi i}-1} d w \\
& +\int_{0}^{-\frac{\pi}{2}} \frac{e^{i \lambda\left(2 \pi i+\varepsilon e^{i \theta}\right)}}{e^{2 \pi i+\varepsilon e^{i \theta}}-1} i \varepsilon e^{i \theta} d \theta-\int_{\varepsilon}^{2 \pi-\varepsilon} \frac{e^{i \lambda(i y)}}{e^{i y}-1} i d y \\
& +\int_{\frac{\pi}{2}}^{0} \frac{e^{i \lambda\left(\varepsilon e^{i \theta}\right)}}{e^{\varepsilon e^{i \theta}}-1} i \varepsilon e^{i \theta} d \theta=0 .
\end{aligned}
$$
in $(3.8)$

Oneobtains the following relationshipif $R \rightarrow+\infty, \varepsilon \rightarrow 0$

$$
\begin{gathered}
\int_{0}^{+\infty} \frac{e^{i \lambda w}}{e^{w}-1} d w-e^{-2 \pi \lambda} \int_{0}^{+\infty} \frac{e^{i \lambda w}}{e^{w}-1} d w+\int_{0}^{-\frac{\pi}{2}} i e^{-2 \pi \lambda} d \theta \\
-i \int_{0}^{2 \pi} \frac{e^{-\lambda y}}{e^{i y}-1} d y+\int_{\frac{\pi}{2}}^{0} i d \theta=0,
\end{gathered}
$$

Consequently, we have

$$
\left(1-e^{-2 \pi \lambda}\right) \int_{0}^{+\infty} \frac{e^{i \lambda w}}{e^{w}-1} d w-i \frac{\pi}{2}\left(1+e^{-2 \pi \lambda}\right)-i \int_{0}^{2 \pi} \frac{e^{-\lambda y}}{e^{i y}-1} d y=0,
$$

The above relationship can be rewritten as following after simple manipulations

$$
\begin{aligned}
\int_{0}^{+\infty} \frac{e^{i \lambda w}}{e^{w}-1} d w= & i \frac{\pi}{2} \frac{1+e^{-2 \pi \lambda}}{1-e^{-2 \pi \lambda}} \\
& +i \frac{1}{1-e^{-2 \pi \lambda}} \int_{0}^{2 \pi} \frac{e^{-\lambda y}(\cos y-1-i \sin y)}{(\cos y-1)^{2}+\sin ^{2} y} d y
\end{aligned}
$$

At this point, it suffices to take real part of the above relationship, to obtain

$$
\int_{0}^{+\infty} \frac{\cos \lambda w}{e^{w}-1} d w=\frac{1}{1-e^{-2 \pi \lambda}} \int_{0}^{2 \pi} \frac{e^{-\lambda y} \sin y}{2(1-\cos y)} d y
$$

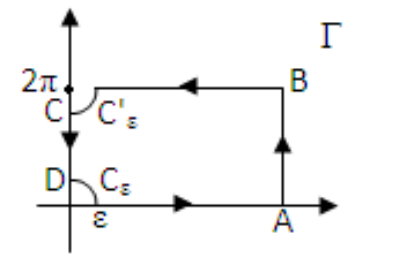

Figure 3.1 differentiating (3.11) with respect to $\lambda$ and then multiplying by $\frac{1}{\lambda}$ we get

$$
\begin{aligned}
& \frac{1}{\lambda} \int_{0}^{+\infty} \frac{w \sin \lambda w}{e^{w}-1} d w \\
& =\frac{-1}{\lambda\left(1-e^{-2 \pi \lambda}\right)^{2}} \int_{0}^{2 \pi} \frac{e^{-\lambda y}\left(e^{-2 \pi \lambda}(y-2 \pi)-y\right) \sin y}{2(1-\cos y)} d y,
\end{aligned}
$$

now, from (3.4) it is clear that

$$
\begin{aligned}
S & =\sum_{n=1}^{\infty} \frac{2 n}{\left(n^{2}+\lambda^{2}\right)^{2}} \\
& =\frac{-1}{\lambda\left(1-e^{-2 \pi \lambda}\right)^{2}} \int_{0}^{2 \pi} \frac{e^{-\lambda y}\left(e^{-2 \pi \lambda}(y-2 \pi)-y\right) \sin y}{2(1-\cos y)} d y,
\end{aligned}
$$

or we may write

$$
S=\frac{1}{2 \lambda\left(1-e^{-2 \pi \lambda}\right)^{2}} \int_{0}^{2 \pi} e^{-\lambda y}\left((2 \pi-y) e^{-2 \pi \lambda}+y\right) \cot \frac{y}{2} d y .
$$

LEMмA 3.2 The following relationship holds true for alternating Mathieu series

$$
\begin{aligned}
\tilde{S} & =\sum_{n=1}^{\infty}(-1)^{n-1} \frac{2 n}{\left(n^{2}+\lambda^{2}\right)^{2}} \\
& =\frac{-1}{2 \lambda\left(1-e^{-2 \pi \lambda}\right)^{2}} \int_{0}^{2 \pi} e^{-\lambda y}\left(e^{-2 \pi \lambda}(y-2 \pi)-y\right) \tan \frac{y}{2} d y .
\end{aligned}
$$

Proof. Similarly to lemma 3.1 from the following integral representation for alternating Mathieu series

$$
\sum_{n=1}^{\infty}(-1)^{n-1} \frac{2 n}{\left(n^{2}+\lambda^{2}\right)^{2}}=\frac{1}{\lambda} \int_{0}^{\infty} \frac{w \sin \lambda w}{e^{w}+1} d w
$$

we have to evaluate

$$
\tilde{I}(\lambda)=\int_{0}^{\infty} \frac{\cos \lambda w}{e^{w}+1} d w
$$

and then differentiate with respect to $\lambda$ to get the desired result.

Let us consider the complex function

$$
g(z)=\frac{e^{i \lambda z}}{e^{z}+1},
$$

with simple pole at point $z=\pi i$. Consider the following integral along the path which is shown in figure 3.2

$$
\frac{1}{2 \pi i} \oint_{\Gamma^{\prime}} g(z) d z=0 .
$$




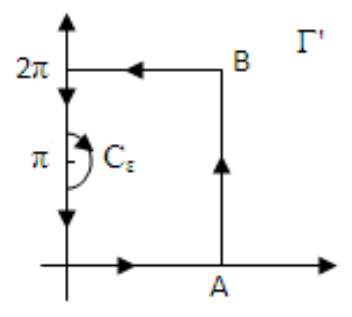

Figure 3.2

Similarly to lemma 3.1 and after performing all the calculations, we get the desired result

$$
\begin{aligned}
\tilde{S} & =\sum_{n=1}^{\infty}(-1)^{n-1} \frac{2 n}{\left(n^{2}+\lambda^{2}\right)^{2}} \\
& =\frac{1}{2 \lambda\left(1-e^{-2 \pi \lambda}\right)^{2}} \int_{0}^{2 \pi} e^{-\lambda y}\left((2 \pi-y) e^{-2 \pi \lambda}+y\right) \tan \frac{y}{2} d y .
\end{aligned}
$$

\section{Generalized Stieltjes and Post Widder Integral Transforms}

Definition 4.1 Generalized Stieltjes transform is defined as follows

$$
F(y)=S_{\rho}\{f(t) ; t \rightarrow y\}=\int_{0}^{\infty} \frac{f(t)}{(t+y)^{\rho}} d t, \quad|\arg y|<\pi
$$

that has as its inverse transform( see $^{12}$ )

$$
S_{\rho}^{-1}\{F(y) ; y \rightarrow t\}=-\frac{1}{2 \pi i} t^{\rho} \int_{C}(1+y)^{\rho-1} F^{\prime}(t y) d y . \quad \rho>0
$$

In the special $\rho=1$ case the transform in the above relation reduces to the Stieltjes transform

$$
S\{f(t) ; t \rightarrow y\}=\int_{0}^{\infty} \frac{f(t)}{t+y} d t
$$

It is well- known that the second iterate of the Laplace transform is Stieltjes transform, that is

$$
L^{2}\{f(t) ; y\}=L\{L\{f(t) ; u\}, y\}=S\{f(t), y\} .
$$

EXAMPLE 4.2 Evaluate Stieltjes transform of the function $\cos (a \sqrt{x})$.

Solution. By definition we have

$$
S\{\cos (a \sqrt{x}) ; x \rightarrow z\}=\int_{0}^{\infty} \frac{\cos (a \sqrt{x})}{x+z} d x
$$

making a change of variables $x=u^{2}$ we get

$$
S\{\cos (a \sqrt{x}) ; x \rightarrow z\}=2 \int_{0}^{\infty} \frac{u}{u^{2}+z} \cos (a u) d u,
$$

by cosine Fourier transform it can be written in the form

$$
S\{\cos (a \sqrt{x}) ; x \rightarrow z\}=2 F_{c}\left\{\frac{u}{u^{2}+z} ; u \rightarrow a\right\}=\frac{1}{4} \pi a^{2} e^{-a \sqrt{z}} .
$$

EXAMPLE 4.3 Solving the following Stieltjes type singular integral equation.

$$
\int_{0}^{\infty} \frac{\varphi(x)}{x+z} d x=\frac{\Gamma\left(1-\frac{1}{n}\right) \Gamma\left(\frac{1}{n}\right)}{\sqrt[n]{z}}
$$

Solution. We know that Stieltjes transform is the second iterate of Laplace transform, so we have

$$
L^{-1}\left\{\Gamma\left(1-\frac{1}{n}\right) \Gamma\left(\frac{1}{n}\right) z^{-\frac{1}{n}} ; z \rightarrow t\right\}=\Gamma\left(1-\frac{1}{n}\right) t^{\frac{1}{n}-1}
$$

and consequently

$$
L^{-1}\left\{\Gamma\left(1-\frac{1}{n}\right) t^{\frac{1}{n}-1} ; t \rightarrow x\right\}=x^{-\frac{1}{n}}
$$

it means that

$$
\varphi(x)=L^{-1}\left\{\frac{\Gamma\left(1-\frac{1}{n}\right) \Gamma\left(\frac{1}{n}\right)}{\sqrt[n]{z}} ; z \rightarrow x\right\}=x^{-\frac{1}{n}}
$$

Difinition 4.4 We define the convolution of two functions $f, g^{14}$

$$
(f \otimes g)(t)=f(t) \int_{0}^{\infty} \frac{g(u)}{u-t} d u+g(t) \int_{0}^{\infty} \frac{f(u)}{u-t} d u,
$$

provided that the integrals exist.

Lemma 4.5 (Convolution) Let $f, g \in L_{1}\left(R_{+}\right)$and let the Stieltjes transform of $f(t) \int_{0}^{\infty} \frac{g(u)}{u-t} d u$ and $g(t) \int_{0}^{\infty} \frac{f(u)}{u-t} d u$ be absolutely convergent. Then there exists the Stieltjes transform of the convolution $f \otimes g$ and it holds that

$$
S(f \otimes g)=S(f) S(g) .
$$

Proof. See ${ }^{14}$. 
EXAMPLE 4.6 Evaluate inverse Stieltjes transform of the function $\frac{1}{\sqrt{s}(s+a)}$, for $a \in R_{-}$.

Solution. By using lemma 4.5 we have

$$
S^{-1}\left\{\frac{1}{\sqrt{s}(s+a)} ; s \rightarrow t\right\}=S^{-1}\left\{\frac{1}{\sqrt{s}}\right\} \otimes S^{-1}\left\{\frac{1}{s+a}\right\},
$$

on the other hand the Stieltjes transform is the second iterate of Laplace transform, so that

$$
S^{-1}\left\{\frac{1}{\sqrt{s}}\right\}=\frac{1}{\pi \sqrt{t}}, S^{-1}\left\{\frac{1}{s+a}\right\}=\delta(t-a),
$$

therefore, the final solution can be written in the form

$$
\begin{aligned}
S^{-1}\left\{\frac{1}{\sqrt{s}(s+a)} ; s \rightarrow t\right\}= & \delta(t-a) \int_{0}^{\infty} \frac{1}{\pi \sqrt{u}(u-t)} d u \\
& +\frac{1}{\pi \sqrt{t}} \int_{0}^{\infty} \frac{\delta(u-a)}{(u-t)} d u .
\end{aligned}
$$

By a change of variables $u^{2}=x$ in the first integral we have

$$
S^{-1}\left\{\frac{1}{\sqrt{s}(s+a)} ; s \rightarrow t\right\}=-\frac{\delta(t-a)}{\sqrt{t}}+\frac{1}{\pi \sqrt{t}(a-t)} .
$$

Definition 4.7 The generalized Post-Widder transform is defined as

$$
P_{\rho}\{f(x) ; x \rightarrow s\}=\int_{0}^{\infty} \frac{x f(x)}{\left(x^{2}+s^{2}\right)^{\rho}} d x .
$$

In the special case $\rho=1$ the transform in (5.3) reduces to the Post-Widder transform

$$
P\{f(x) ; x \rightarrow s\}=\int_{0}^{\infty} \frac{x f(x)}{x^{2}+s^{2}} d x .
$$

EXAMPLE 4.8 Evaluate Post Widder transform of the function $x \sqrt{x}$.

Solution. By definition we have

$$
P\{x \sqrt{x} ; x \rightarrow s\}=\int_{0}^{\infty} \frac{x^{\frac{5}{2}}}{\left(x^{2}+s^{2}\right)} d x,
$$

By a change of variables $\frac{x}{s}=u$ we have

$$
P\{x \sqrt{x} ; x \rightarrow s\}=s^{\frac{3}{2}} \int_{0}^{\infty} \frac{u^{\frac{5}{2}}}{\left(u^{2}+1\right)} d u
$$

it can be considered as Mellin transform of the function $\frac{-u}{\left(u^{2}+1\right)} M\left\{\frac{-u}{\left(u^{2}+1\right)} ; u \rightarrow p=\frac{5}{2}\right\}$.

Let us define the function $f(u)=\operatorname{arccot} u$ then it is clear that $P\{x \sqrt{x}\}=-s^{\frac{3}{2}} M\left\{u f^{\prime}(u) ; u \rightarrow p=\frac{5}{2}\right\}$ which is $\frac{5}{2} s^{\frac{3}{2}} M\left\{\operatorname{arccot} u ; u \rightarrow p=\frac{5}{2}\right\}$, therefore the final result is

$$
P\{x \sqrt{x} ; x \rightarrow s\}=\frac{\pi}{2} \sqrt{2} s^{\frac{3}{2}}
$$

Lемма 4.9 The inverse of generalized Post-Widder transform is as following

$$
f(x)=P_{\rho}^{-1}\{F(s) ; s \rightarrow x\}=-\frac{x^{2 \rho}}{\pi i} \oint_{\Gamma}(1+s)^{\rho-1} F^{\prime}\left(x^{2} s\right) d s .
$$

Proof. By definition we have

$$
E(\rho, s)=P_{\rho}\{f(x) ; x \rightarrow s\}=\int_{0}^{\infty} \frac{x f(x)}{\left(x^{2}+s^{2}\right)^{\rho}} d x,
$$

by a change of variables $x^{2}=u, s^{2}=w$ the above equation can be rewritten in the form

$$
F(\rho, w)=\int_{0}^{\infty} \frac{f(\sqrt{u})}{2(u+w)^{\rho}} d u
$$

this is a generalized Stieltjes transform which inverse is

$$
\frac{1}{2} f(\sqrt{u})=-\frac{u^{\rho}}{2 \pi i} \oint_{\Gamma}(1+w)^{\rho-1} F^{\prime}(u w) d w,
$$

where $\Gamma$ is a closed contour containing origin. Now substitute $x^{2}=u, s^{2}=w$ to obtain

$$
\begin{aligned}
f(x) & =P_{\rho}^{-1}\{F(s) ; s \rightarrow x\} \\
& =-\frac{2 x^{2 \rho}}{\pi i} \oint_{\Gamma} s\left(1+s^{2}\right)^{\rho-1} F^{\prime}\left(x^{2} s^{2}\right) d s .
\end{aligned}
$$

For the special case $\rho=1$ the inverse of Post-Widder transform is in the form

$$
P^{-1}\{F(s) ; s \rightarrow x\}=-\frac{2 x^{2}}{\pi i} \oint_{\Gamma} s F^{\prime}\left(x^{2} s^{2}\right) d s .
$$

Corollary 4.10 The following relationship holds true

$$
f(x)=P^{-1}\{F(s) ; s \rightarrow x\}=\frac{1}{\pi i}\left\{F\left(x e^{-i \pi}\right)-F\left(x e^{i \pi}\right)\right\} .
$$


Proof. By using lemma 4.9 we have

$$
P^{-1}\{F(s) ; s \rightarrow x\}=-\frac{2 x^{2}}{\pi i} \oint_{\Gamma} s F^{\prime}\left(x^{2} s^{2}\right) d s,
$$

it suffices to make a change of variables $F\left(x^{2} s^{2}\right)=u$ to get

$$
P^{-1}\{F(s) ; s \rightarrow x\}=-\frac{1}{\pi i} \oint_{\Gamma} d\left(F\left(x^{2} s^{2}\right)\right) .
$$

where $\Gamma$ is a contour containing origin that could be chosen, for instance, to be unit circle.

Note that it is not really a closed contour because $F(s)$ has a branch cut along the negative real axis $\left(\right.$ see $\left.^{12}\right)$.

Therefore, we have the result as follows

$$
P^{-1}\{F(s) ; s \rightarrow x\}=\frac{1}{\pi i}\left\{F\left(x^{2} e^{-i \pi}\right)-F\left(x^{2} e^{i \pi}\right)\right\} .
$$

LeMmA 4.11 Let us assume that $S\{f(x) ; x \rightarrow s\}=F(s)$, then we have the following relationship

$$
f(x)=\frac{1}{2 \pi i}\left\{F\left(x e^{-i \pi}\right)-F\left(x e^{i \pi}\right)\right\} .
$$

Proof. By definition we know that

$$
S^{-1}\{F(s) ; s \rightarrow x\}=-\frac{s}{2 \pi i} \int_{C} F^{\prime}(s w) d w
$$

by a change of variables $F(s w)=\eta$, the following equation will be in the form

$$
S^{-1}\{F(s) ; s \rightarrow x\}=-\frac{1}{2 \pi i} \int_{C} d \eta,
$$

where $\mathrm{C}$ is a contour containing origin that has a branch cut along the negative real axis.

Therefore we have the result as follows

$$
S^{-1}\{F(s) ; s \rightarrow x\}=\frac{1}{2 \pi i}\left\{F\left(x e^{-i \pi}\right)-F\left(x e^{i \pi}\right)\right\} .
$$

LEMмA 4.12 The following relationship holds true

$$
\begin{aligned}
& P\left\{\frac{(\operatorname{bei}(2 \sqrt{x}))^{2}}{x} ; x \rightarrow \lambda\right\} \\
& =\frac{\pi}{4 \lambda} I_{0}(2 \sqrt{\lambda})\left[I_{0}(2 \sqrt{\lambda})+J_{0}(2 \sqrt{\lambda})\right] .
\end{aligned}
$$

Proof. Define the function

$$
I(\eta, \mu)=\int_{0}^{\infty} \frac{b e i(2 \sqrt{\eta x}) b e i(2 \sqrt{\mu x})}{x^{2}+\lambda^{2}} d x,
$$

now by taking two dimensional Laplace transform with respect to $\eta, \mu$ we have

$$
\begin{aligned}
& L_{2}\{I(\eta, \mu) ; \eta \rightarrow p, \mu \rightarrow q\} \\
& =\frac{1}{p q} \int_{0}^{\infty} \frac{\cos \frac{x}{p} \cos \frac{x}{q}}{x^{2}+\lambda^{2}} d x \\
& =\frac{1}{2 p q} \int_{0}^{\infty} \frac{\cos \left(\frac{x}{p}-\frac{x}{q}\right)+\cos \left(\frac{x}{p}+\frac{x}{q}\right)}{x^{2}+\lambda^{2}} d x .
\end{aligned}
$$

On the other hand by using the following integral relationship

$$
\int_{0}^{\infty} \frac{\cos m x}{x^{2}+\lambda^{2}} d x=\frac{\pi}{2 \lambda} e^{-m \lambda},
$$

and substituting in (4.13) we obtain

$$
L_{2}\{I(\eta, \mu)\}=\frac{\pi e^{-\frac{\lambda}{p}}}{2 p q \lambda} \cosh \frac{\lambda}{q} .
$$

By taking inverse two dimensional Laplace transform we can write

$$
\begin{aligned}
I(\eta, \mu) & =L_{2}^{-1}\left\{\frac{\pi e^{-\frac{\lambda}{p}}}{2 p q \lambda} \cosh \frac{\lambda}{q}\right\} \\
& =\frac{\pi}{2 \lambda} \times \frac{1}{2 \pi i} \int_{c-i \infty}^{c+i \infty} \frac{e^{-\frac{\lambda}{p}}}{p}\left(\frac{1}{2 \pi i} \int_{c^{\prime}-i \infty}^{c^{\prime}+i \infty} \frac{\cosh \frac{\lambda}{q}}{q} e^{q \mu} d q\right) e^{p \eta} d p,
\end{aligned}
$$

from table of inverse Laplace transforms we get ( see ${ }^{15}$ )

$$
=\frac{\pi}{4 \lambda} I_{0}(2 \sqrt{\lambda \eta})\left[I_{0}(2 \sqrt{\lambda \mu})+J_{0}(2 \sqrt{\lambda \mu})\right],
$$

now it suffices to let $\eta=\mu=1$ to get the desired relationship as below

$$
\begin{aligned}
P\left\{\frac{(b e i(2 \sqrt{x}))^{2}}{x} ;\right. & x \rightarrow \lambda\}=\int_{0}^{\infty} \frac{(b e i(2 \sqrt{x}))^{2}}{x^{2}+\lambda^{2}} d x \\
& =\frac{\pi}{4 \lambda} I_{0}(2 \sqrt{\lambda})\left[I_{0}(2 \sqrt{\lambda})+J_{0}(2 \sqrt{\lambda})\right] .
\end{aligned}
$$

Lemma 4.13 (Convolution for Post-Widder transform) Let $f, g \in L_{1}\left(R_{+}\right)$and let the Stieltjes transform of $f(\sqrt{t}) \int_{0}^{\infty} \frac{g(u)}{(u-\sqrt{t})} d u$ and $g(\sqrt{t}) \int_{0}^{\infty} \frac{f(u)}{(u-\sqrt{t})} d u$ be absolutely 
convergent. Then there exists the Post-Widder transform of the convolution $f \otimes g$ and it holds that

$$
P\{f(t) \otimes g(t)\}=2 P(f) P(g)
$$

Proof. By definition we know that

$$
P\{f(t)\}=\left\{\int_{0}^{\infty} \frac{t f(t)}{t^{2}+s^{2}} d t\right\}=\frac{1}{2} S\left\{f(\sqrt{u}) ; u \rightarrow s^{2}\right\},
$$

(making a change of variables $t^{2}=u$ ). So it can be followed that

$$
P\{f(t) \otimes g(t)\}=\frac{1}{2} S\left\{f(\sqrt{u}) \otimes g(\sqrt{u}) ; u \rightarrow s^{2}\right\},
$$

now from lemma 4.4 we get the following relation

$$
P\{f(t) \otimes g(t)\}=\frac{1}{2} S\left\{f(\sqrt{u}) ; u \rightarrow s^{2}\right\} S\left\{g(\sqrt{u}) ; u \rightarrow s^{2}\right\},
$$

again from (4.14) we obtain

$$
\begin{aligned}
P\{f(t) \otimes g(t)\} & =\frac{1}{2}(2 P\{f(t) ; t \rightarrow s\})(2 P\{g(t) ; t \rightarrow s\}) \\
& =2 P(f) P(g) .
\end{aligned}
$$

EXAMPLE 4.14 Evaluate the inverse Post Widder transform of the function $H(s)=\frac{s \sqrt{s}}{s+a}$.

Solution. By using lemma 4.13, let us consider two functions $F(s)=s \sqrt{s}, G(s)=\frac{1}{s+a}$, then we have

$$
P^{-1}\{H(s) ; s \rightarrow x\}=P^{-1}\{F(s)\} \otimes P^{-1}\{G(s)\},
$$

but performing some manipulations we obtain

$$
P^{-1}\{F(s)\}=\frac{\sqrt{2}}{\pi} x \sqrt{x}, \quad P^{-1}\{G(s)\}=\frac{2}{\pi} \frac{x}{x^{2}+a^{2}},
$$

therefore from lemma 4.13 we get the following relationship

$$
\begin{aligned}
P^{-1}\{H(s) ; s \rightarrow x\}= & \frac{\sqrt{2} x^{\frac{1}{4}}}{2 \pi^{2}} \int_{0}^{\infty} \frac{d u}{\left(u+a^{2}\right)(u-x)} \\
& +\frac{\sqrt{2}}{2(x+a)} \int_{0}^{\infty} \frac{u^{\frac{1}{4}}}{(u-x)} d u,
\end{aligned}
$$

accordingly, the final result is obtained as

$$
P^{-1}\{H(s) ; s \rightarrow x\}=-\frac{\sqrt{2} x^{\frac{1}{4}}}{2 \pi^{2}} \frac{\log \left(\frac{x}{a^{2}}\right)}{x+a^{2}}-\frac{\pi x^{\frac{5}{4}}}{(x+a)} .
$$

\section{Main Result}

In this section, we implement joint Laplace - Fourier sine transform for solving certain fractional wave equation for vibration of a wire of finite length.

Problem 5.1 Consider the following fractional PDE which describes the vibrations of a wire of length $b$ with both ends fixed which at point $x=b$ has been moved up for $\varepsilon$ and released

$$
\frac{\partial^{2 \alpha} u}{\partial t^{2 \alpha}}=c^{2} \frac{\partial^{2} u}{\partial x^{2}} \quad, \quad 0 \leq \alpha \leq 1
$$

with boundary and initial conditions

$$
\begin{aligned}
& u(0, t)=u(L, t)=0, \\
& u_{t}(x, 0)=0, \\
& u(x, 0)= \begin{cases}\frac{\varepsilon x}{b} \quad ; 0<x<b \\
\frac{\varepsilon(x-L)}{b-L} & ; b<x<L\end{cases}
\end{aligned}
$$

Solution. Taking finite Fourier sine transform of (5.1) we have

$$
\int_{0}^{L} \frac{\partial^{2 \alpha} u}{\partial t^{2 \alpha}} \sin \frac{n \pi x}{L} d x=c^{2} \int_{0}^{L} \frac{\partial^{2} u}{\partial x^{2}} \sin \frac{n \pi x}{L} d x
$$

now by integrating by parts we get

$$
F_{s, x}\left\{\frac{\partial^{2} u}{\partial x^{2}}\right\}=\int_{0}^{L} \frac{\partial^{2} u}{\partial x^{2}} \sin \frac{n \pi x}{L} d x=-\left(\frac{n \pi}{L}\right)^{2} F_{s, x}\{u(x, t)\} .
$$

Substituting in (5.1) one will obtain the following relationship

$$
\frac{\partial^{2 \alpha}}{\partial t^{2 \alpha}} U(n, t)+\frac{c^{2} n^{2} \pi^{2}}{L^{2}} U(n, t)=0
$$

in which $U(n, t)=F_{s, x}\{u(x, t)\}$. Now take Laplace transform of the above equation with respect to $t$

$$
s^{2 \alpha} \bar{U}(n, s)-\frac{\varepsilon L^{3}}{n^{2} \pi^{2} b(L-b)} \sin \frac{n \pi b}{L}+\frac{c^{2} n^{2} \pi^{2}}{L^{2}} \bar{U}(n, s)=0,
$$

in which $\bar{U}(n, s)=L\{U(n, t) ; t \rightarrow s\}$. Therefore 


$$
\bar{U}(n, s)=\frac{\varepsilon L^{3}}{n^{2} \pi^{2} b(L-b)} \sin \frac{n \pi b}{L}\left(\frac{1}{s^{2 \alpha}+\frac{c^{2} n^{2} \pi^{2}}{L^{2}}}\right)
$$

by using Schouten-Vanderpol theorem we have

$$
\begin{aligned}
& L^{-1}\left\{\frac{1}{s^{2 \alpha}+\frac{c^{2} n^{2} \pi^{2}}{L^{2}}} ; s \rightarrow t\right\} \\
& =\frac{1}{2 \pi i} \int_{0}^{\infty} e^{-t \eta}\left\{\frac{1}{\eta^{2 \alpha} e^{2 i \alpha \pi}+\frac{c^{2} n^{2} \pi^{2}}{L^{2}}}-\frac{1}{\eta^{2 \alpha} e^{-2 i \alpha \pi}+\frac{c^{2} n^{2} \pi^{2}}{L^{2}}}\right\} d \eta,
\end{aligned}
$$

hence, the solution is as follows

$$
\begin{aligned}
u(x, t)= & \frac{1}{L \pi i} \sum_{j=1}^{\infty} \sin \frac{n \pi x}{L} \int_{0}^{\infty} e^{-t \eta}\left\{\frac{1}{\eta^{2 \alpha} e^{2 i \alpha \pi}+\frac{c^{2} n^{2} \pi^{2}}{L^{2}}}\right. \\
& \left.-\frac{1}{\eta^{2 \alpha} e^{-2 i \alpha \pi}+\frac{c^{2} n^{2} \pi^{2}}{L^{2}}}\right\} d \eta .
\end{aligned}
$$

\section{Conclusion}

The paper is devoted to study applications of one and two dimensional Laplace, finite Fourier, sine transform, generalized Stieltjes and Post-Widder transforms in details and their applications. Integral transforms provides a powerful method for analyzing linear systems. The authors also discussed Mathieu series-and introduced new integral representations for the above mentioned series.

\section{References}

1. Aghili A, Zeinali H. Integral transform method for solving Volterra singular integral equations and non homogenous time fractional PDEs. Gen Math Notes. 2013 Jan; 14(1):6-20.
2. Aghili A, Zeinali H. Integral transform methods for solving fractional PDEs and evaluation of certain integrals and series. Intern Journal of Physics and Mathematical Sciences. 2012; 2(4).

3. Aghili A, Moghaddam BS. Laplace transform pairs of $\mathrm{n}$ - dimensions and a wave equation. Intern Math Journal. 2004; 5(4); 377-82.

4. Aghili A, Moghaddam BS. Multi-dimensional Laplace transform and systems of partial differential equations. Intern Math Journal. 2006; 1(6):21-24.

5. Aghili A, Moghaddam BS. Laplace transform pairs of $\mathrm{N}$ - dimensions and second order linear differential equations with constant coefficients. Annales Mathematicae et informaticae. 2008; 35:3-10.

6. Dahiya RS, Saberi-Nadjafi J. Theorems on N-dimensional Laplace Transforms and their applications. 15th Annual ConferenceofAppliedMathematics,Univ.ofCentralOklahoma. Electronic Journal of Diferential Equations, Conference 02. 1999; 61-74.

7. Dahiya RS, Vinayagamoorthy M. Laplace transfom pairs of $\mathrm{n}$ - dimensions and heat conduction problem. Math Comput Modelling. 1990; 13(10):35-50.

8. Ditkin VA, Prudnikov AP. Operational calculus in two variables and its application.New Yourk: Pergamon Press; 1962.

9. Podlubny I. Fractional differential equations. San Diego, CA: Academic Press; 1999.

10. Bell WW. Special functions for scientists and engineers, DVan Nostrand Company LTD, Canada; 1968.

11. Elezovic N, Srivastava HM, Tomovski Z. Integral representations and integral transforms of some families of Mathieu type series. Integral transforms and special functions. 2008; $1: 1-15$

12. Schwarz JH. The generalized Stieljes transform and its inverse. J Math Phys. 2005; 46; 013501.

13. Duffy DG. Transform methods for solving partial differential equations. New Youk: Chapman and Hall/CRC; 2004.

14. Glaeske HJ, Prudnikov AP, Skornik KA. Operational Calculus and Related Topics. USA: Chapman and Hall/ CRC; 2006.

15. Polyanin AD, Manzhirov AV. Handbook of Integral Equations. USA:L Chapman and Hall/CRC; 2008.

16. Bobylev AV, Cercignani C. The inverse Laplace transform of some analytic functions with an application to the eternal solutions of the Boltzmann equation. Applied Mathematics Letters. 2002; 15:807-13. 